\title{
The effects of femoral component design on the patello-femoral joint in a PS total knee arthroplasty
}

\author{
Pier Francesco Indelli • Massimiliano Marcucci • \\ Gennaro Pipino · Sophie Charlton · Christian Carulli • \\ Massimo Innocenti
}

Received: 30 April 2013

(C) Springer-Verlag Berlin Heidelberg 2013

\begin{abstract}
Introduction Anterior knee pain following TKA performed utilizing the PFC Sigma system still represents a cause of failure. The purpose of this study was to evaluate whether or not a recent change in the femoral design (PFC Sigma PS) had a positive impact on the patello-femoral complication rate.

Materials and methods A consecutive series of 100 TKA using the PFC Sigma PS system was followed prospectively for a minimum of 3 years. All patellae were replaced and a standard lateral release was never performed. Radiographic analysis following the Knee Society Score (KSS) included antero-posterior weight-bearing, lateral and bilateral axial radiographs. TKA rotational alignment was recorded at the final follow-up in 30 consecutive knees by performing a CT evaluation.

Results Good to excellent clinical results according to the KSS were achieved in $94 \%$ of the knees. Survival

\author{
P. F. Indelli · M. Marcucci \\ Centro Eccellenza Sostituzioni Articolari Toscana (CESAT), \\ Clinica Ortopedica Universita' di Firenze, Florence, Italy \\ P. F. Indelli · M. Marcucci \\ Fondazione Onlus, “...In Cammino...”, Fucecchio, Italy \\ P. F. Indelli $\cdot$ S. Charlton \\ Breyer Center for Overseas Study, Stanford University \\ in Florence, Florence, Italy \\ P. F. Indelli · M. Marcucci · C. Carulli · M. Innocenti \\ Clinica Ortopedica Universita' di Firenze, Florence, Italy \\ P. F. Indelli $(\varangle) \cdot$ M. Marcucci \\ CESAT, Piazza Lavagnini 1, 50054 Fucecchio, Firenze, Italy \\ e-mail: pierfrancesco.indelli@unifi.it \\ G. Pipino \\ Ospedale Villa Regina, Bologna, Italy
}

without need of reoperation for any reason was $98 \%$ at 3 years minimum follow-up; two reoperations were done for removal of fibromatous intra-articular tissue ("Clunk syndrome"). There were no revisions for septic or aseptic loosening of the components. The mean ROM improved from $104^{\circ}$ preoperatively to $115^{\circ}\left(97^{\circ}-132^{\circ}\right)$ postoperatively: postoperative flexion was $120^{\circ}$ or more in $58 \%$ of the knees. Severe anterior knee pain was present in $9 \%$ of patients. Radiographic evaluation showed 90 knees with a tibio-femoral anatomical axis between $8^{\circ}$ and $2^{\circ}$ of valgus $\left( \pm 3^{\circ}\right.$ from the intraoperative goal). CT evaluation of 30 consecutive knees showed that the femoral component positioning in relationship to the trans-epicondylar axis had only $2.80^{\circ}$ of external rotation $\left( \pm 2.10^{\circ}\right)$ with respect to a planned external rotation of $3^{\circ}$. This difference was statistically significant.

Conclusions Although the PFC Sigma PS system provides good and predictable results for tricompartmental arthritis of the knee, anterior mechanism complications still represent a reason for dissatisfaction in a substantial group of patients.

Keywords Total knee arthroplasty $\cdot$ Patella $\cdot$ Anterior knee pain $\cdot$ Femoral component design $\cdot$ Patellar resurfacing $\cdot$ TKA

\section{Introduction}

Although current results are relatively promising, total knee arthroplasty (TKA) is not a "perfect" operation. The Ontario Joint Registry showed that only $70 \%$ of patients met their expectations at 1 year follow-up after TKA [1]. Twenty percent of patients report persistent knee pain at 5 years after surgery [2], where anterior knee pain related 
to the patello-femoral joint represents an often cited cause for revision surgery [3]. Historically, this complication has been linked to component design characterized by an "unfriendly" trochlea: in fact, basic design modifications in the same TKA implant were related to a substantial decrease in extensor mechanism complications [4]. In more recent times, the implant industry's answer to patient and surgeon dissatisfaction has been to produce gender-specific designs [5] and more anatomical femoral components. On the other side, tibiofemoral malalignment with respect to the mechanical axis and rotational malalignment have been associated with implant failure and increase in patello-femoral complication rate $[6,7]$.

The PFC Sigma (DePuy Orthopaedics Inc., Warsaw, USA) TKA was introduced in 1996 as a development of the Press-Fit Condylar (PFC) implant (Johnson \& Johnson, Raynham, Massachusetts, USA). Design features included modularity to increase intraoperative adaptability, an updated femoral coronal geometry and a deeper and more prolonged trochlear groove to improve patellar tracking beyond $90^{\circ}$ of flexion. In recent years, a number of studies have investigated the functional outcome of the PFC and PFC Sigma knee systems [8-10], showing satisfactory midterm results. Unfortunately, both painless and painful patellar crepitations, general anterior knee pain and instances of patellar clunk syndrome, have been reported with an incidence of up to $21 \%$ by many authors, including the implant's designers [11, 12]. Because of these and other issues on patello-femoral complications, the PFC Sigma femoral component was re-designed, becoming available in 2009 under the name PFC Sigma PS (DePuy Orthopaedics Inc., Warsaw, USA). The new principal design features included a "J curve" femoral design, three different tangential radius curves in the sagittal profile, and a single radius curve in the coronal profiles. The femoral box edge and the trochlear groove edges were refined too, to provide a better patellar route during ROM. A new specific TKA instrumentation (High Performance: HP; DePuy Orthopaedics Inc., Warsaw, USA) was introduced as well.

The aim of this study was to investigate whether these modifications to the femoral design, coupled with the new instrumentation, had a positive impact on avoiding the development of anterior knee pain and other extensor mechanism complications. We have undertaken a 3-year minimum follow-up of the new TKA system and have studied the frequency of complications and reasons for patient dissatisfaction. To our knowledge, this is the first clinical and radiological study on the PFC Sigma PS TKA implant.

\section{Materials and methods}

Between October 2009 and April 2010, 100 consecutive PFC Sigma PS posterior stabilized fixed-bearing

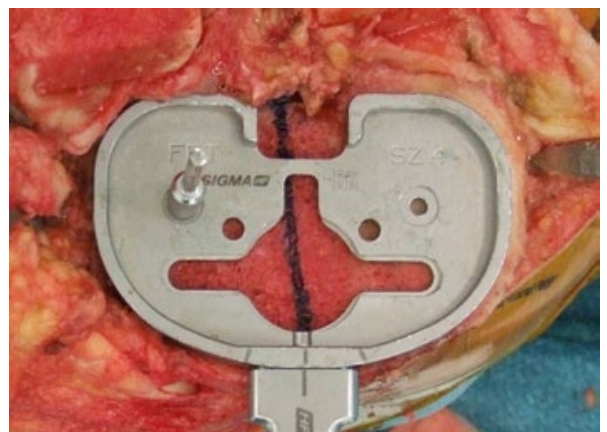

Fig. 1 Right knee. The rotational reference landmark for the tibial component was always the anterior tibial cortex ("curve-on-curve" technique). The "Akagi line" is highlighted: the tibial component is externally rotated with respect to this anatomical landmark

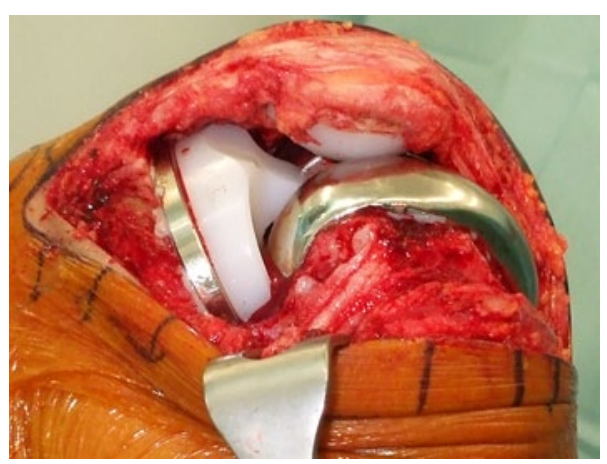

Fig. 2 Right knee. Patellar tracking in deep flexion showing full congruence in the trochlear groove

knee prostheses were performed on 100 consecutive, nonselected patients after approval from the institutional review board (IRB). All patients gave their informed consent prior to their inclusion in the study. The indications for surgery were advanced degenerative change with severe pain on weight-bearing, impaired knee function and limitation of daily activity. The preoperative diagnosis in this consecutive series was always osteoarthritis. The patients' mean age at surgery was 73 years (range 55-87 years). There were 67 females and 33 males in the study group. Patients' preoperative Knee Society Score (KSS) and Knee Society functional score (KSFS) were recorded. The surgical approach included a standard midline skin incision and a medial peripatellar capsulotomy, consistently avoiding standard lateral releases. The Sigma HP instrumentation was used to allow $3^{\circ}$ of external rotation to the cemented femoral component and the "balanced gaps technique" was the chosen surgical technique. The tibial component rotational alignment was set matching the tibial anterior cortex (Fig. 1) with its anterior side ("curve-on-curve technique" for rotational alignment) [13]. The desired tibio-femoral AP axis was set at $5^{\circ}$ of valgus, which has been shown to be safe in primary TKA 
[14]. All patellae were replaced with a so-called "freehand technique" independently from the gravity of patellofemoral osteoarthritis, and tracking of the patella was checked using the "no thumb technique" (Fig. 2): a release of the deep lateral patello-femoral ligament was performed at the end of the procedure in $17 \%$ of patients to gain better patellar tracking. Any sagittal osteotomy of the lateral patellar facet was performed in this consecutive series.

All patients were prospectively followed up for a minimum of 3 years, with a mean follow-up of 44 months. At the final follow-up, all patients completed the Western Ontario and McMaster Universities Osteoarthritis Index (WOMAC) questionnaire. Full-length leg radiograph, standard antero-posterior weight-bearing, and lateral and bilateral axial radiographs were performed on all patients during the final follow-up. All radiographs were reviewed by a blinded viewer (SC) according to the Knee Society criteria for radiolucency, change in the position of the components, femorotibial alignment, and evidence for loosening, wear and osteolysis. TKA rotational alignment was recorded at follow-up in 30 consecutive knees (number 30 to number 60) by performing a CT evaluation. This radiological evaluation was limited to only 30 TKA on direct request by our IRB. This study sub-group was composed of 22 females and 8 males: their average age was 69 years (range 52-83 years). The knee deformity in this sub-group of patients was varus in 25 knees (range $6^{\circ}-17^{\circ}$ ) and valgus in $5\left(\right.$ range $5^{\circ}-14^{\circ}$ ). The right knee was involved in 23 cases and the left in 7 . The CT study was performed [13] with the patient assuming a supine position on the radiological table with the knee bent at $20^{\circ}$; CT images were $2 \mathrm{~mm}$ in thickness and with $2 \mathrm{~mm}$ in reconstructive increments, starting at the distal metaphysis and finishing at the tibial tubercle. Particular attention was paid to the following CT measurements, both preoperatively and at the 3-year follow-up: patellar tilt (PTA) according to Fulkerson et al. [15], patellar congruence angle (CA) according to Aglietti et al. [16], patellar lateralization measuring the lateral patellar displacement (LPD) according to Brossman et al. [17] and femoral component external rotation in relation to the trans-epicondylar axis (TEA), measuring the posterior condyles angle (PCA) according to Boisgard et al. [18].

All statistics and calculations were performed with SPSS 16.0 (SPSS Inc., Chicago, IL). Two-tailed $P$ values $<0.05$ were considered to be statistically significant.

\section{Results}

Clinical results

At the final follow-up, all patients were available for analysis. Good to excellent clinical results according to the
Knee Society Rating System were achieved in $94 \%$ of the knees: the clinical evaluation of the 100 consecutive knees showed a mean KSS of 89 points and a mean KSFS of 76 points. The mean preoperative KSS was 39 points (range 30-65), where the mean preoperative KSFS was 36 points (range 25-55). The mean ROM improved from $104^{\circ}$ preoperatively to $115^{\circ}\left(97^{\circ}-132^{\circ}\right)$ postoperatively: postoperative flexion was $120^{\circ}$ or more in $58 \%$ of knees. Average WOMAC at the follow-up was 32 (range 22-107). Loss of full extension at follow-up more than $5^{\circ}$ was present in three knees ( $3 \%$ ); preoperative loss of full extension was present in 73 knees $(73 \%)$, averaging $13^{\circ}$ (range $1^{\circ}-27^{\circ}$ ).

\section{Radiological results}

The radiological assessment at follow-up showed that the femorotibial alignment, with respect to a desired anatomical axis of $5^{\circ}$ of valgus, averaged $5.3^{\circ}$ of valgus (range from $6^{\circ}$ of valgus to $4^{\circ}$ of varus). Ninety knees had a tibiofemoral anatomical axis between $8^{\circ}$ and $2^{\circ}$ of valgus $\left( \pm 3^{\circ}\right.$ from the goal). On the Knee Society TKA roentgenographic evaluation form, radiolucencies were found around the femoral components in three $(3 \%)$ cases: all of them were behind the prosthetic anterior flange. Tibial tray radiolucent lines were found in 4 of the 100 knees $(4 \%)$ : they were all in zone 1 and 2 in the AP knee view, none was more than $1 \mathrm{~mm}$ in width and none was progressive. None of the components was found to be radiologically loose. No radiolucent lines were found in all-polyethylene patellae.

All 30 patients selected for CT evaluation were available at follow-up and the results are shown in a different study [13]. The rotational alignment of the femoral component has been studied measuring the posterior condyles angle (PCA): the femoral component positioning in relationship to the trans-epicondylar axis at follow-up showed an average of $2.80^{\circ}$ of external rotation $\left( \pm 2.10^{\circ}\right)$. The planned external rotation of the femoral component was $3^{\circ}$.

\section{Postoperative complications}

There were no intraoperative complications. In the early postoperative period, one patient experienced a partial rupture of the quadricipital tendon during a session of physical therapy: because the extensor mechanism was overall intact, the lesion was treated conservatively. ROM at final follow-up in this patient was $0^{\circ}-122^{\circ}$. Anterior knee pain was present in nine patients (9\% of the knees). Severe and painful crepitations were noted in five of these patients (5\%), two of whom $(2 \%)$ required reoperation to remove fibromatous intra-articular tissue ("Clunk syndrome") (Fig. 3). Both patients had a CT scan evaluation showing slight internal rotation of the femoral component $\left(2.5^{\circ}\right.$ and $2.8^{\circ}$, respectively). One patient had a traumatic patellar 


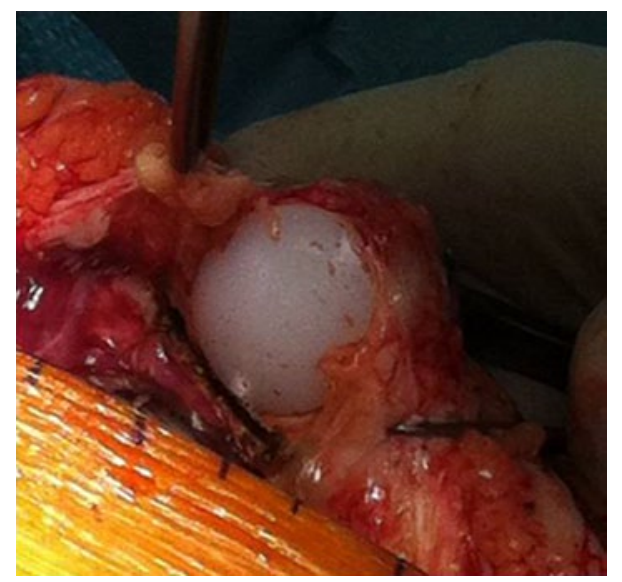

Fig. 3 Left knee. Removal of fibromatous intra-articular tissue ("Clunk syndrome")

fracture at 14 months from the index procedure. The fracture was treated conservatively, but the patient reported severe anterior knee pain at follow-up. This patient was in the CT study group too: the femoral component positioning in relationship to the trans-epicondylar axis at followup showed only $2.60^{\circ}$ of external rotation. The other three patients in this group had mild to moderate painful crepitations during daily activities, but were not part of the CT study sub-group, so the authors were not able to investigate a possible rotational malalignment of the components.

\section{Discussion}

The purpose of this study was to evaluate whether or not a change in the femoral design of a widely used PS TKA (PFC Sigma PS) had a positive impact on the extensor mechanism complication rate. The results of the Press-Fit Condylar (PFC) TKA, improvement of the total condylar knee implant, were overall satisfactory with a $93 \%$ survival rate at 15 years [18]. Unfortunately, the revision rate for patello-femoral related problems was reported up to $5.2 \%$ [19]. The PFC Sigma implant was first introduced in 1996, providing an updated femoral coronal geometry and a deeper trochlear groove to improve patellar tracking with respect to previous similar implants. Painless or painful patellar crepitations up to $21 \%$ and patellar clunk syndromes up to $5 \%$ have been reported by many authors, including the implant's designers [11, 12]. Revisions for patello-femoral problems have shown to still only be a minor issue with this implant [20], not differing between patient's sex [21]. The use of the mobile-bearing PFC Sigma option did not reduce the patello-femoral complication rate, as it failed to improve patellar tracking [22]. In fact, Meftah et al. recently reported a $21 \%$ incidence of anterior knee pain and crepitation at 3-5 years [23]. Previous studies hypothesized that this high rate of patellofemoral complications might be linked to the medialization of the prosthetic femoral groove typical of this implant [24]. In a different study, Meijerink et al. [25] noted a trend toward lateral tracking of the patella in early flexion when using the PFC Sigma prosthesis. Because of these issues, a new PFC Sigma PS System (De Puy, Warsaw, USA) was introduced in 2009, with new instrumentation and a peculiar femoral design. This new design followed many recommendations from the literature: Barink et al. [26] showed that an appropriate design for the prosthetic trochlea rather than its replacement was the main determinant of good patella-femoral outcomes in TKA; Kulkarni et al. [27] showed that a femoral "J curve design" allowed for patella medialization, thus reducing patellofemoral lateral shear forces; D'Lima et al. [28] demonstrated that a single radius curve in the femoral coronal profile restored quadriceps force to physiological levels during ROM. In fact, the new PFC Sigma PS has a "J curve" shape with three different tangential radii in the sagittal profile and a single radius curve in the coronal profile. A prolonged anterior flange and a "smoother" transition from trochlea to the box are innovative to this design, as well.

The primary objective of the current study was to evaluate if recent improvements in the PFC Sigma PS femoral design correlated with higher patient satisfaction, particularly with regard to anterior knee pain and symptomatic crepitations. Anterior knee pain was present in $9 \%$ of our knees, severe painful crepitations was noted in $5 \%$ of these patients and two patients $(2 \%)$ required reoperation with removal of fibromatous intra-articular tissue ("Clunk Syndrome"). Slight internal rotation of the femoral component was highlighted as the main predisposing factor of this complication. In fact, the femoral component positioning in relationship to the trans-epicondylar axis in 30 consecutive knees showed an average of $2.80^{\circ}$ of external rotation with respect to a planned $3^{\circ}$, and both patients who underwent "Clunk syndrome" removal showed insufficient femoral component external rotation. Previous reports suggested that the commonly reported $3^{\circ}$ of external rotation of the femoral component might be insufficient to make a rectangular flexion gap at $90^{\circ}$, increasing the risk of patello-femoral complications [29]. Nonetheless, the rate of extensor mechanism complication registered in the current study, still considered high by the authors, diminished sensibly with respect to previous reports [23]. This study confirmed good to excellent results with the PFC PS Sigma implant in $94 \%$ of the patients. The mean ROM improved from $104^{\circ}$ preoperatively to $115^{\circ}$ at the final follow-up.

The radiological evaluation of the implant alignment showed a correct restoration of the anatomical axis $\pm 3^{\circ}$ in $90 \%$ of the knees. Conversely, Winemaker et al. [30] 
recently showed no association between component position and early failure in 398 primary cemented TKA at 14 years follow-up. It was suggested that restoring a straight axis in TKA might aim at the wrong target. The still high incidence of extensor mechanism complications typical of the current study might be related to the fact that patellar tracking is governed by a combination of static and dynamic factors. Maltracking may result from excessive or unbalanced tension in the surrounding soft tissues during knee flexion, so our static radiographic measurements do not reflect patello-femoral congruence during ROM, leaving the question on the effect of the femoral component design on anterior knee pain development partially unanswered. This is a limitation of our study.

The present study confirmed that the reduction of major extensor mechanism complications with respect to the previous version of the PFC Sigma TKA might be strictly linked to the surgical technique during patellar replacement, as shown by others [31, 32]. In particular, meticulous removal of the peri-patellar synovial layer is mandatory to avoid postoperative clunk syndrome.

This study had several other limitations. First, it was performed in a select patient population affected by knee osteoarthritis and selection bias may have influenced the clinical outcomes. Also, this assessment lacks both a comparison group with the previous version of the identical prosthesis and longer-term follow-up.

Despite overall positive results in the first three postoperative years, the PFC Sigma PS TKA system still presents a high rate of extensor mechanism complications. The surgical community has hence become divided on the issue of how the patella is best served when performing TKA [33, 34]. The results presented in the current study showed that the design changes to the original PFC Sigma femoral component leading to the PFC Sigma PS system reduced, but did not abolish the incidence of postoperative anterior knee pain.

\section{References}

1. Bourne RB, Chesworth BM, Davis AM, Mahomed NN, Charron KD (2010) Patient satisfaction after total knee arthroplasty: who is satisfied and who is not? Clin Orthop Relat Res 468(1):57-63

2. Hofmann S, Seitlinger G, Djahani O, Pietsch M (2011) The painful knee after TKA: a diagnostic algorithm for failure analysis. Knee Surg Sp Traumatol Arthrosc 19(9):1442-1452

3. Patel J, Ries MD, Bozic KJ (2008) Extensor mechanism complications after total knee arthroplasty. Instr Course Lect 2008(57):283-294

4. Aglietti P, Baldini A, Buzzi R, Indelli PF (2001) Patella resurfacing in TKA: functional evaluation and complications. Knee Surg Sp Traumatol Arthrosc 9(Suppl 1):27-33

5. Kim YH, Choi Y, Kim JS (2010) Comparison of a standard and a gender-specific posterior cruciate-substituting high-flexion knee prosthesis: a prospective, randomized, short-term outcome study. J Bone Joint Surg Am 92(10):1911-1920
6. Zihlmann MS, Stacoff A, Romero J, Quervain IK, Stüssi E (2005) Biomechanical background and clinical observations of rotational malalignment in TKA: literature review and consequences. Clin Biomech 20(7):661-668

7. Benjamin J (2006) Component alignment in total knee arthroplasty. Instr Course Lect 2006(55):405-412

8. Dalury DF, Gonzales RA, Adams MJ, Gruen TA, Trier K (2008) Midterm results with the PFC Sigma total knee arthroplasty system. J Arthroplast 23(2):175-181

9. Zaki SH, Rafiq I, Kapoor A, Raut V, Gambhir AK, Porter ML (2007) Medium term results with the Press Fit Condylar (PFC) Sigma knee prosthesis the Wrightington experience. Acta Orthop Belg 73:55-59

10. Hanusch B, Lou TN, Warriner G, Hui A, Gregg P (2010) Functional outcome of PFC Sigma fixed and rotating-platform total knee arthroplasty. A prospective randomized controlled trial. Int Orthop 34:349-354

11. Ranawat AS, Ranawat CS, Slamin JE, Dennis DA (2006) Patellar crepitation in the P.F.C. sigma total knee system. Orthopedics 29(9 Suppl):68-70

12. Meftah M, Ranawat AS, Ranawat CS (2011) Safety and efficacy of a rotating-platform, high-flexion knee design three- to fiveyear follow-up. J Arthroplasty 27(2):201-206

13. Indelli PF, Marcucci M, Cariello D, Poli P, Innocenti M (2012) Contemporary femoral designs in total knee arthroplasty: effects on the patello-femoral congruence. Int Orthop 36(6):1167-1173

14. Kharwadkar N, Kent RE, Sharara KH, Naique S (2006) $5^{\circ}$ to $6^{\circ}$ of distal femoral cut for uncomplicated primary total knee arthroplasty: is it safe? Knee 13(1):57-60

15. Fulkerson JP, Schutzer SF, Ramsby GR, Bernstein RA (1987) Computerized tomography of the patellofemoral joint before and after lateral release or realignment. Arthroscopy 3(1):19-24

16. Aglietti P, Insall JN, Cerulli G (1983) Patellar pain and incongruence: I. Measurements of incongruence. Clin Orthop Relat Res 176:217-224

17. Brossmann J, Muhle C, Schröder C, Melchert UH et al (1993) Patellar tracking patterns during active and passive knee extension: evolution with motion-triggered. Cine MR Imaging Radiol 187(1):205-212

18. Boisgard S, Moreau PE, Descamps S, Courtalhiac C, Silbert H, Moreel P, Michel JL, Levai JP (2003) Computed tomographic study of the posterior condylar angle in arthritic knees: its use in the rotational positioning of the femoral implant of total knee prostheses. Surg Radiol Anat 25:330-334

19. Ranawat CS, Rose HA, Rich DS (1984) Total condylar knee arthroplasty for valgus and combined valgus-flexion deformity of the knee. Instr Course Lect 198433:412-416

20. Rodricks DJ, Patil S, Pulido P, Colwell CW Jr (2007) Press-fit condylar design total knee arthroplasty fourteen to seventeenyear follow-up. J Bone Joint Surg Am 89(1):89-95

21. Dalury DF, Barrett WP, Mason JB, Goldstein WM et al (2008) Midterm survival of a contemporary modular knee replacement. A multicentre study of 1970 knees. JBJS (Br) 90-B:1594-1596

22. Dalury DF, Mason JB, Murphy JA, Adams MJ (2009) Analysis of the outcome in male and female patients using a unisex total knee replacement system. JBJS (Br) 91-B:357-360

23. Pagnano MV, Trousdale RT (2004) Rotating platform knees did not improve patellar tracking: a prospective, randomized study of 240 primary TKA. Clin Orthop Relat Res 428:221-227

24. Meftah M, Ranawat AS, Ranawat CS (2011) The natural history of anterior knee pain in 2 posterior-stabilized, modular total knee arthroplasty designs. J Arthroplasty 26(8):1145-1148

25. Meijerink HJ, Barink M, Van Loon CJ et al (2007) The trochlea is medialized by total knee arthroplasty: an intraoperative assessment in 61 patients. Acta Ortop 78(1):123-127

26. Barink M, Van de Groes S, Verdonschot $\mathrm{N}$ et al (2006) The difference in trochlear orientation between the natural knee and current 
prosthetic knee designs: towards a truly physiological prosthetic groove orientation. J Biomech 39:1708-1715

27. Kulkarni SK, Freeman MAR, Poal-Manresa JC, Asencio JI, Rodriquez JJ (2000) The patellofemoral joint in total knee arthroplasty. Is the design the critical factor? J Arthroplasty 15(4):424-429

28. D'Lima DD, Steklov N, Patil S, Colwell CW (2008) The Mark Coventry Award: in vivo knee forces during recreation and exercise after knee arthroplasty. Clin Orthop Relat Res 466(11):2605-2611

29. Ostermeier S, Hurschler C, Stukenborg-Colsman C (2004) Quadriceps function after TKA — an in vitro study in a knee kinematic simulator. Clin Biomech 19(3):270-276

30. Winemaker MJ (2002) Perfect balance in total knee arthroplasty: the elusive compromise. J Arthroplasty 17(1):2-10
31. Parratte S, Pagnano MW, Trousdale RT, Berry DJ (2010) Effect of postoperative mechanical axis alignment on the fifteen-year survival of modern, cemented total knee replacements. J Bone Joint Surg Am 92(12):2143-2149

32. Lachiewicz PF (2004) Implant design and techniques for patellar resurfacing in total knee arthroplasty. Instr Course Lect 2004(53):187-191

33. Chonko DJ, Lombardi AV Jr, Berend KR (2004) Patella baja and total knee arthroplasty (TKA): etiology, diagnosis, and management. Surg Technol Int 12:231-238

34. Schindler OS (2012) The controversy of patellar resurfacing in TKA: ibisne in medio tutissimus? Knee Surg Sp Traumatol Arthrosc 20:1227-1244 